\title{
A Method for Determining the Flory Theta Temperature and Entropy Parameter of Single- or Multicomponent- Polymer-Single Solvent Systems from the Critical Solution Point Data
}

\author{
Kenji KAMIDE and Shigenobu MATSUDA \\ Textile Research Laboratory, Asahi Chemical Industry Co., Ltd., \\ 11-7, Hacchonawate, Takatsuki, Osaka 569, Japan
}

(Received April 11, 1984)

\begin{abstract}
An attempt was made to determine the concentration dependence coefficients, $p_{1}$ and $p_{2}$, of the polymer-solvent interaction parameter $\chi\left[\chi=\chi_{0}\left(1+p_{1} v_{\mathrm{p}}+p_{2} v_{\mathrm{p}}^{2}\right) ; \chi_{0}\right.$; concentration independent coefficient, $v_{\mathrm{p}}$; polymer-volume fraction] from the critical solution concentration $v_{\mathrm{p}}^{\mathrm{c}}$ and the weight- and $z$-average relative molar volume ratios of the polymer to the solvent, $X_{w}$ and $X_{z}$, of polymer samples in multicomponent polymer-single solvent systems and evaluate the Flory's theta solution temperature $\theta$ and entropy parameter $\psi$ for a system using the critical solution temperature $T_{\mathrm{c}}$ and critical $\chi_{0}$ parameter $\chi_{0}^{\mathrm{c}}$. For this purpose, a theory is proposed to calculate $\chi_{0}^{\mathrm{c}}$ from $p_{1}, p_{2}, X_{w}$, and $X_{z}$. The method was applied to the literature data of upper and lower critical solution point (UCSP and LCSP) regions for polystyrene (PS)-cyclohexane (CH) and PS-methylcyclohexane systems. The values of $p_{1}$ and $p_{2}$, thus obtained for UCSP of PS-CH system, were in good agreement with those estimated by Kamide et al. from the cloud point curve. The $\theta$ and $\psi$ values obtained are compared with those by the Koningsveld et al. method using the same critical point data and with those by the second virial coefficient method.

KEY WORDS Concentration Dependence Parameter / Polymer-Solvent Interaction Parameter / Critical Solution Temperature / Entropy Parameter / Multicomponent Polymer-Solvent System / Polystyrene /
\end{abstract}

The Flory temperature $\theta$ and entropy parameter $\psi$ were experimentally determined from the temperature dependence of the second virial coefficient $A_{2}(\theta$ as the temperature at which $A_{2}$ becomes zero and $\psi$ from the temperature dependence of $A_{2}$ (see eq 25) at $\theta$, respectively) and the critical point (the temperature $T_{\mathrm{c}}$ and the volume fraction $v_{\mathrm{p}}^{\mathrm{c}}$ ), extrapolating by the Shultz-Flory ${ }^{2}$ method for a polymer of infinite molecular weight (see eq $\left.21^{\prime}\right)$. Although being only approximate, the Shultz-Flory method has found rather widely use. For all polymer-solvent systems investigated, it was confirmed that $\theta$ temperatures evaluated by the above two procedures agree fairly well with each other, but $\psi$ values estimated by the second virial coefficient method are significantly smaller than those obtained by the critical point method. ${ }^{3}$ Stockmayer $^{4}$ pointed out the inadequancy of the basic equation (eq $21^{\prime}$ ) of the Shultz-Flory theory and proposed an alternative equation (eq 18), rigorously derived for polydisperse polymer solutions. Another factor is the concentration dependence of the $\chi$-parameter, which was completely neglected in the Shultz-Flory and Stockmayer methods. To solve this problem, Koningsveld et al. ${ }^{12}$ estimated a method for estimating $\theta$ and $\psi$ from data on critical concentration $v_{\mathrm{p}}^{\mathrm{c}}$ and critical temperature $T_{\mathrm{c}}$ for a series of solutions differing in average molecular weight. They employed a pair interaction parameters $g$, expanding as a series function of the polymer volume fraction $v_{\mathrm{p}}$ in 
the form,

$$
g=\sum_{i=0}^{n} g_{i} v_{\mathrm{p}}^{i}
$$

Here they assumed that $g_{0}$ could be devided into temperature-independent and -dependent components given by

$$
g_{0}=g_{00}+g_{01} / T
$$

The $\psi$ and $\theta$ can be calculated from

$$
\begin{gathered}
\psi=\frac{1}{2}-g_{00}-g_{1} \\
\theta=g_{01} / \psi
\end{gathered}
$$

Note that the concentration- and temperaturedependence of the $g$ parameter, in other words, the parameters $g_{00}, g_{01}, g_{1}$, and $g_{2}$ were evaluated so that the deviation of the experimental $v_{\mathrm{p}}^{\mathrm{c}}$ and $T_{\mathrm{c}}$ data for all samples from the theory would be minimum. Koningsveld et al.'s procedure was applied to polystyrene (PS)-cyclohexane (CH) and PS-methylcyclohexane $(\mathrm{MCH})$ and the $\psi$ values were calculated. In their method, the curve fitting method was repeatedly used to determine $g_{i}$ (for example, see Figures 1 and 2). The concentration dependence of the $g$ parameter (accordingly, $\chi$-parameter) can also be determined not only from the critical point, but by various other methods such as osmotic pressure, light scattering, ultracentrifuge, vapor pressure, and phase separation, by which the $\chi$-parameter is directly obtained.

In this article, we have attempted to clarify whether and to what extent $\psi$ and $\theta$ are influenced by the concentration dependence of the $\chi$-parameter and polymolecularity of the samples and propose a method for estimating $\psi$ and $\theta$ from $T_{\mathrm{c}}$ alone, taking into account the above two factors determined independently by other methods, without assuming a specific temperature dependence of the $\chi$-parameter, as in the case of using experimental data from the literature, we applied our method to polystyrene solutions for a comparison with the results obtained by other method, such as those of Shultz-Flory, ${ }^{2}$ Stockmayer, ${ }^{4}$ Koningsveld et al., ${ }^{8,12}$ and the second virial coefficient method.

\section{THEORETICAL BACKGROUND}

The $\chi$-parameter can be completely phenomelogically expressed in a power series of the concentration, ${ }^{5}$

$$
\begin{aligned}
\chi & =\sum_{j=0}^{n} \chi_{i} v_{\mathrm{p}}^{j} \\
& =\chi_{0}\left(1+\sum_{j=1}^{n} p_{j} v_{\mathrm{p}}^{j}\right)
\end{aligned}
$$

for multicomponent polymer-single solvent systems. Here, $\chi_{0}$ is a parameter independent of concentration, and $p_{j}$, the concentrationdependence parameter. Kamide et al. ${ }^{6,7}$ showed from the phase separation experiment for polystyrene in cyclohexane and methylcyclohexane that $\chi_{0}$ is empirically given by

$\chi_{0}=\chi_{00}\left(1+k_{0}(1-\theta / T) / X_{n}\right)$

where $\chi_{00}$ is a parameter independent of the ratio of the molar volume of the polymer (i.e., $X$-mer) to that of the solvent $X_{i}, X_{n}$; number average of $X_{i}, k_{0}$; parameter independent of temperature and $X_{i}$, and $T$; the temperature. The applicability of eq 6 has not yet been widely confirmed by actual experiments, ${ }^{6,7}$ and the molecular weight dependence of $\chi$, expressed by eq 6 is not considered. $\chi, \chi_{0}, p_{i}$, and $P_{n}$ are directly related to $g, g_{i}$, and $g_{n}$ through the relations,

$$
\begin{aligned}
& \chi=g-\left(1-v_{\mathrm{p}}\right)\left(\frac{\partial g}{\partial v_{\mathrm{p}}}\right) \\
& \chi_{0}=g_{0}-g_{1} \\
& p_{i}=(i+1) \frac{g_{i}-g_{i+1}}{g_{0}-g_{1}} \\
& p_{n}=(n+1) \frac{g_{n}}{g_{0}-g_{1}}
\end{aligned}
$$

According to the Koningsveld theory, $p_{i}$ is a 
function of temperature through $g_{0}$. An alternative expression of the concentrationdependence of $g$ was proposed by Koningsveld et al. ${ }^{8}$ as

$$
g=\alpha+\frac{\beta}{1-\gamma v_{\mathrm{p}}}
$$

with

$$
\beta=\beta_{00}+\beta_{01} / T
$$

Here, $\alpha, \beta_{00}, \beta_{01}$, and $\gamma$ are constants for a given polymer/solvent system. If $0<\gamma<1$ holds, $0<\gamma v_{\mathrm{p}}<1$ can be obtained and the second term in the right-hand side of eq 11 expanded as a series function

$$
\begin{aligned}
g & =\alpha+\beta+\sum_{i=1}^{\infty} \beta\left(\gamma v_{\mathrm{p}}\right)^{i} \\
& \simeq \alpha+\beta+\sum_{i=1}^{n} \beta\left(\gamma v_{\mathrm{p}}\right)^{i}
\end{aligned}
$$

Equation 13 reduces to eq 1 by putting $\alpha+\beta=$ $g_{0}$ and $\beta \gamma^{i}=g_{i}(i=1, \cdots, n)$. In this case, $\chi_{0}$ and $p_{i}$ can be expressed in terms of $\alpha, \beta$, and $\gamma$ by

$$
\begin{gathered}
\chi_{0}=\alpha+\beta(1-\gamma) \\
p_{i}=(i+1) \frac{\beta \gamma^{i}(1-\gamma)}{\alpha+\beta(1-\gamma)} \quad(i=1, \cdots, n-1) \\
p_{n}=(n+1) \frac{\beta \gamma^{n}}{\alpha+\beta(1-\gamma)}
\end{gathered}
$$

Note that $P_{i}$ is sometimes temperature dependent due to the temperature dependence of $\alpha, \beta$, and $\gamma$. At the critical point, the following equations can be derived, ${ }^{9}$

$$
\frac{1}{X_{w} v_{\mathrm{p}}^{\mathrm{c}}}+\frac{1}{1-v_{\mathrm{p}}^{\mathrm{c}}}-\chi_{0}^{\mathrm{c}}\left(2+\sum_{j=1}^{n} p_{j}(j+2) v_{\mathrm{p}}^{\mathrm{c}^{j}}\right)=0
$$

and

$\frac{1}{\left(1-v_{\mathrm{p}}^{\mathrm{c}}\right)^{2}}-\frac{X_{z}}{\left(X_{w} v_{\mathrm{p}}^{\mathrm{c}}\right)^{2}}-\chi_{0}^{\mathrm{c}} \sum_{j=1}^{n} p_{j} j(j+2) v_{\mathrm{p}}^{\mathrm{c}^{j-1}}=0$

where $\chi_{0}^{\mathrm{c}}$ is the critical $\chi_{0}, X_{w}$, the weight- average $X$, and $X_{z}$, the $z$-average $X$.

Both $\chi_{0}^{\mathrm{c}}$ and $v_{\mathrm{p}}^{\mathrm{c}}$ can be obtained concurrently through application of eq 14 and 15, using a numerical method, to the data of $X_{w}$, $X_{z}$, and $p_{j}(j=1,2) . \chi_{0}^{\mathrm{c}}$ is related to $T_{\mathrm{c}}, \psi, \theta$ through the relation ${ }^{1,5}$

$$
\frac{1}{T_{\mathrm{c}}}=\frac{\chi_{0}^{\mathrm{c}}}{\theta \psi}+\frac{1}{\theta}\left(1-\frac{1}{2 \psi}\right)
$$

with

$\psi \equiv 1 / 2-\chi_{0, \mathrm{~s}}, \quad \theta \equiv \chi_{0, \mathrm{~h}} /\left(1 / 2-\chi_{0, \mathrm{~s}}\right)$

$\chi_{0, \mathrm{~s}}$ and $\chi_{0, \mathrm{~h}}$ are the entropy and enthalpy components of $\chi_{0}$, respectively (i.e., $\chi_{0}=\chi_{0, \mathrm{~s}}+$ $\left.\chi_{0, \mathrm{~h}}\right)$. Using $\chi_{0}^{\mathrm{c}}$, calculated from eq 14 and 15 , and experimental $T_{\mathrm{c}}$, we can determine $\theta$ and $\psi$ from the plot of $1 / T_{\mathrm{c}}$ against $\chi_{0}^{\mathrm{c}}$. This method is hereafter simply referred to as the KamideMatsuda method.

Putting $p_{j}=0$ (for $j=1, \cdots, n$ ) in eq 14 , we obtain

$$
\chi_{0}^{\mathrm{c}}=\frac{1}{2}\left(\frac{1}{X_{w} v_{\mathrm{p}}^{\mathrm{c}}}+\frac{1}{1-v_{\mathrm{p}}^{\mathrm{c}}}\right)
$$

Substitution of the Stockmayer equation which was derived rigorously for a multicomponent polymers-single solvent system, assuming $p_{j}=0(j=1, \cdots, n)$,

$$
v_{\mathrm{p}}^{\mathrm{c}}=\frac{1}{1+X_{w} / X_{z}^{1 / 2}}
$$

into eq 18 yields

$$
\chi_{0}^{\mathrm{c}}=\frac{1}{2}\left(\frac{X_{z}^{1 / 2}}{X_{w}}+\frac{1}{X_{w}}\right)\left(\frac{X_{w}}{X_{z}^{1 / 2}}+1\right)
$$

Combining eq 16 with eq 20 , we obtain

$$
\begin{aligned}
\frac{1}{T_{\mathrm{c}}}= & \frac{1}{\theta \psi}\left\{\frac{1}{2}\left(\frac{1}{X_{w}^{1 / 2}}+\left(\frac{X_{z}}{X_{w}}\right)^{1 / 2}\right)\right. \\
& \left.\times\left(\frac{1}{X_{w}^{1 / 2}}+\left(\frac{X_{w}}{X_{z}}\right)^{1 / 2}\right)\right\}+\frac{1}{\theta}\left(1-\frac{1}{2 \psi}\right)
\end{aligned}
$$

When $X_{w}=X_{z}$ is assumed, eq 19 and 21 reduce to the well-known equations derived by Shultz and Flory. ${ }^{2}$ 


$$
v_{\mathrm{p}}^{\mathrm{c}}=\frac{1}{1+X_{w}^{1 / 2}}
$$

and

$$
\begin{aligned}
\frac{1}{T_{\mathrm{c}}} & =\frac{1}{\theta \psi}\left(\frac{1}{2}\left(\frac{1}{X_{w}^{1 / 2}}+1\right)^{2}\right)+\frac{1}{\theta}\left(1-\frac{1}{2 \psi}\right) \\
& =\frac{1}{\theta \psi}\left(\frac{1}{X_{w}^{1 / 2}}+\frac{1}{2 X_{w}}\right)+\frac{1}{\theta}
\end{aligned}
$$

Note that eq $21^{\prime}$, is strictly valid only for a monodisperse polymer-single-solvent system, in which the $\chi$-parameter is assumed to be independent of the molecular weight of the polymer and concentration. When eq 6 is applicable, eq 14 and 15 can be modified as,

$$
\begin{aligned}
& \frac{1}{X_{w} v_{\mathrm{p}}^{\mathrm{c}}}+\frac{1}{1-v_{\mathrm{p}}^{\mathrm{c}}}-\chi_{00}^{\mathrm{c}}\left(1+k\left(1-\frac{\theta}{T_{\mathrm{c}}}\right)\right. \\
&\left.\times\left(1+\frac{1}{X_{n}}-\frac{X_{w}}{X_{n}}\right)\right) \\
& \times\left(2+\sum_{j=1}^{n} p_{j}(j+2) v_{\mathrm{p}}^{\mathrm{c}^{j}}\right)=0 \\
& \frac{1}{\left(1-v_{\mathrm{p}}^{\mathrm{c}}\right)^{2}}-\frac{X_{z}}{\left(X_{w} v_{\mathrm{p}}^{\mathrm{c}}\right)^{2}}-\chi_{00}^{\mathrm{c}}\left(1+k_{0}\left(1-\frac{\theta}{T_{\mathrm{c}}}\right)\right. \\
&\left.\times\left(1+\frac{1}{X_{n}}-\frac{X_{w}}{X_{n}}\right)\right) \\
& \times\left(\sum_{j=1}^{n} p_{j} j(j+2) v_{\mathrm{p}}^{\mathrm{c}^{j-1}}\right)=0
\end{aligned}
$$

In the Koningsveld et al. method, $g_{1}$ and $g_{2}$ in eq 1 are calculated from $v_{\mathrm{p}}^{\mathrm{c}}, X_{w}$ and $X_{z}$ by applying the curve fitting method to the equation

$$
\begin{aligned}
Y & \equiv g_{1}-g_{2}+4 g_{2} v_{\mathrm{p}}^{\mathrm{c}} \\
& =\frac{1}{6}\left(\frac{1}{\left(1-v_{\mathrm{p}}^{\mathrm{c}}\right)^{2}}-\frac{X_{z}}{\left(X_{w} v_{\mathrm{p}}^{\mathrm{c}}\right)^{2}}\right)
\end{aligned}
$$

of neutral equilibrium conditions. $g_{0}$ is evaluated from $g_{1}$ and $g_{2}$, thus determined and $v_{\mathrm{p}}^{\mathrm{c}}$ and $X_{w}$, using the spinodal equation,

$$
\begin{aligned}
2 g_{0}= & \frac{1}{1-v_{\mathrm{p}}^{\mathrm{c}}}+\frac{1}{v_{\mathrm{p}}^{\mathrm{c}} X_{w}} \\
& +2 g_{1}\left(1-3 v_{\mathrm{p}}^{\mathrm{c}}\right)+6 g_{2}\left(1-2 v_{\mathrm{p}}^{\mathrm{c}}\right) v_{\mathrm{p}}^{\mathrm{c}}
\end{aligned}
$$

Equation 22 is basically equivalent to eq 15 and eq 23 is the same as eq 14. $g_{00}$ and $g_{01}$ can be estimated from the plot of $\dot{g}_{0} v s .1 / T_{\mathrm{c}}$ according to eq 2 . Replacing $\chi_{0}^{\mathrm{c}}$ with $g_{0}-g_{1}$ in eq 16, eq 3 and 4 can be derived.

Whether the concentration dependence of the $\chi$-parameter and polymolecularity of the polymer should be taken into account to explain the critical point and, in the former case what would be the most reasonable values of $p_{1}, p_{2}, \cdots, p_{n}$, can be decided by comparing the experimental $v_{\mathrm{p}}^{\mathrm{c}}\left(v_{\mathrm{p}}^{\mathrm{c}}(\exp )\right)$ and theoretical $v_{\mathrm{p}}^{\mathrm{c}}\left(v_{\mathrm{p}}^{\mathrm{c}}\right.$ (theo)) data calculated using eq 14,15 (Kamide-Matsuda), eq 19 (Stockmayer), and eq 19' (Shultz-Flory). An analysis of the data on $v_{\mathrm{p}}^{\mathrm{c}}(\exp ), X_{z}$, and $X_{w}$ would provide the most ideal values of $p_{1}, p_{2}, \cdots$, and $p_{n}$ as a combination yielding minimum $\delta$, defined by

$$
\begin{aligned}
& \delta=\sum_{i=1}^{N_{0}}\left(v_{\mathrm{p}}^{\mathrm{c}}(\exp )-v_{\mathrm{p}}^{\mathrm{c}}(\text { theo })\right)_{i}^{2} / N_{0} \\
& \left(N_{0}, \text { total number of samples }\right) .
\end{aligned}
$$

That is, an appropriate choice of $p_{1}, \cdots, p_{\mathbf{n}}, v_{\mathrm{p}}^{\mathrm{c}}$ (theo) will fit $v_{\mathrm{p}}^{\mathrm{c}}(\mathrm{exp})$ for an entire set of data. If $v_{\mathrm{p}}^{\mathrm{c}}(\mathrm{exp})$ and experimental $T_{\mathrm{c}}\left(T_{\mathrm{c}}(\exp )\right)$ data are available for a polymer-solvent system, we can determine $p_{1}, \cdots, p_{\dot{n}}$ from the plot of $v_{\mathrm{p}}^{\mathrm{c}}(\exp ) v s . v_{\mathrm{p}}^{\mathrm{c}}($ theo $)$ and $\theta$ and $\psi$ from the $1 / T_{\mathrm{c}}-\chi_{0}^{\mathrm{c}}$ plot concurrently.

Table I presents the features of the various methods proposed so far for estimating $\theta$ and $\psi$ from critical point data.

\section{APPLICATION TO EXPERIMENTAL DATA}

\section{a) Atactic Polystyrene(PS)-Cyclohexane ( $\mathrm{CH})$}

For PS-CH solutions, Kamide et al. determined $p_{1}=0.6$ and $p_{2}=0$ from a phase equilibrium experiment ${ }^{10}$ and $p_{1}=0.643$ and 
A Method for Determining $\theta$ and $\psi$ from the CSP Data

Table I. Various methods for estimating $\theta$ and $\psi$ from critical point data

\begin{tabular}{lcccc}
\hline \multicolumn{1}{c}{ Method } & \multicolumn{2}{c}{ Parameter prerequisite for applying method } & Parameters deduced \\
\cline { 2 - 4 } & $\begin{array}{c}\text { Molecular } \\
\text { characteristics }\end{array}$ & $\begin{array}{c}\text { Critical } \\
\text { point data }\end{array}$ & $\begin{array}{c}\text { Concentration } \\
\text { dependence of } \chi\end{array}$ & $\begin{array}{c}\text { from the mod } \\
\text { poinn }\end{array}$ \\
\hline Schulz-Flory (eq 20') & $X_{w}$ & $T_{\mathrm{c}}$ & - & $\theta, \psi, v_{\mathrm{p}}^{\mathrm{c}}$ \\
Stockmayer (eq 20) & $X_{w}, X_{z}$ & $T_{\mathrm{c}}$ & - & $\theta, \psi, v_{\mathrm{p}}^{\mathrm{c}}$ \\
Koningsveld et al. (eq 3, 4) & $X_{w}, X_{z}$ & $T_{\mathrm{c}}, v_{\mathrm{p}}^{\mathrm{c}}$ & - & $\theta, \psi, g_{00}, g_{01}, g_{1}, g_{2}$ \\
Kamide-Matsuda (eq 14-16) & $X_{w}, X_{z}$ & $T_{\mathrm{c}}$ & $p_{1}, p_{2}$ & $\theta, \psi, v_{\mathrm{p}}^{\mathrm{c}}, \chi_{00}$ \\
\hline
\end{tabular}

$p_{2}=0.200$ from a cloud point experiment. ${ }^{9}$ The $p_{1}$ and $p_{2}$ values of this system have been evaluated by other workers by osmotic pressure, ${ }^{11}$ isothermal distillation, ${ }^{11}$ vapor pressure, ${ }^{11}$ critical solution point, ${ }^{12,13}$ and ultracentrifuge. ${ }^{14}$ For example, Koningsveld and his coworkers ${ }^{12}$ analyzed critical solution data using their procedure to obtain $\alpha=-0.1597$, $\beta_{00}=0.4987, \beta_{01}=111.74$, and $\gamma=0.2365$ (accordingly, $p_{1}=0.623$ and $p_{2}=0.290$ at $307 \mathrm{~K}$ ). Kuwahara et al. ${ }^{13}$ obtained $p_{1}=0.607$ and $p_{2}=0.512$ at $299 \mathrm{~K}$ from critical points by the Koningsveld et al. method.

Literature data on the upper critical solution points (UCSP) ( $v_{\mathrm{p}}^{\mathrm{c}}$ and $T_{\mathrm{c}}$ ) of Koningsveld $e t$ $a l .{ }^{12}$ and of Kuwahara and his collaborators $^{13,15}$ and on lower critical solution points (LCSP) of Saeki et al. ${ }^{15}$ are available for determining $p_{1}$, and $p_{2}$, using Koningsveld's and our methods. Here, since Kuwahara et al. and Saeki et al. evaluated only $X_{w}$ and $X_{n}$ for these samples, we estimated $X_{z}$ assuming the Schulz-Zimm type molecular weight distribution for all PS samples.

Figure 1 shows a plot of $\mathrm{Y}$ in eq 22 versus $v_{\mathrm{p}}^{\mathrm{c}}$ for the literature data on the UCSP and LCSP of a $\mathrm{PS} / \mathrm{CH}$ system. From this figure, we estimated, using the least-square regression method, $g_{1}$ to be 0.214 and $g_{2}, 0.057$ for UCSP and $g_{1}=0.1345$ and $g_{2}=0.007_{9}$ for LCSP. The plot for LCSP is very far from linearity because experimental techniques become much more difficult, thus limiting the accuracy of the $g_{1}$ and $g_{2}$ values for LCSP. $g_{0}$ was calculated by putting the $g_{1}$ and $g_{2}$ values thus obtained

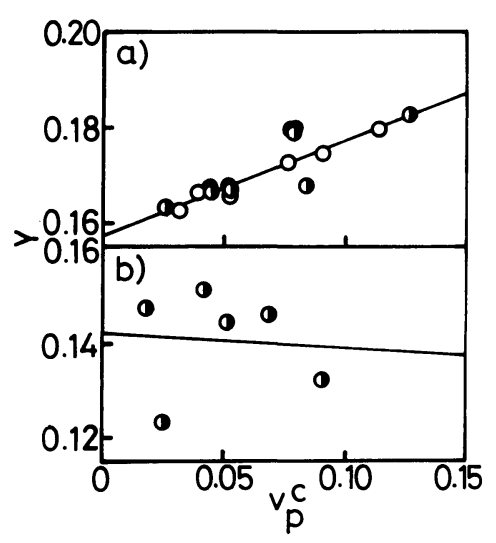

Figure 1. Plot of $Y$ in eq 21 against $v_{\mathrm{p}}^{\mathrm{c}}$ for the $\operatorname{UCSP}(\mathrm{a})$ and LCSP (b) of the polystyrene/cyclohexane system. $\bigcirc$, Koningsveld et al. ${ }^{12} ; \bigcirc$, Kuwahara et al. ${ }^{13} ; \bigcirc$, Saeki et $a .^{15}$

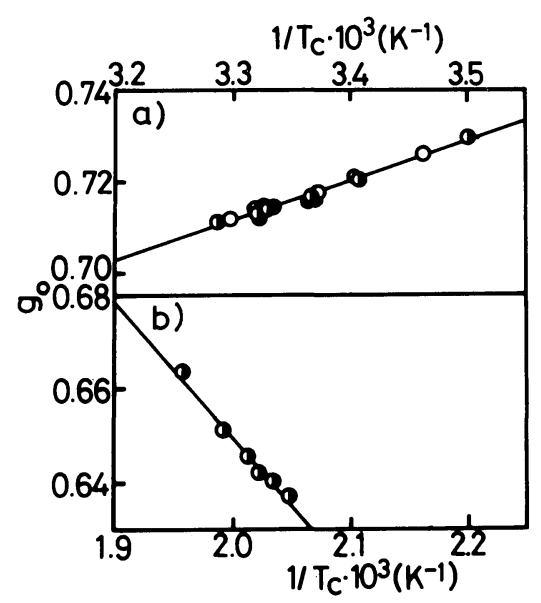

Figure 2. Plot of $g_{0}$ in eq 22 versus $1 / T_{\mathrm{c}}$ for the UCSP (a) and LCSP (b) of the polystyrene/cyclohexane system. O., Koningsveld et al. ${ }^{12} ; \mathbf{O}$, Kuwahara et al. ${ }^{13} ; \boldsymbol{O}$, Saeki et al. ${ }^{15}$ 
Table II. Flory $\theta$ temperature and entropy parameter $\psi$ of the upper solution critical point for the polystyrene-cyclohexane system

\begin{tabular}{|c|c|c|c|c|c|}
\hline \multicolumn{2}{|c|}{ Method } & \multicolumn{2}{|c|}{$\begin{array}{c}\text { Concentration } \\
\text { dependence of } \chi\end{array}$} & \multirow[t]{2}{*}{$\theta / K$} & \multirow[t]{2}{*}{$\psi$} \\
\hline & & $p_{1}$ & $p_{2}$ & & \\
\hline \multirow{8}{*}{$\begin{array}{l}\text { Critical } \\
\text { point }\end{array}$} & Shultz-Flory (eq 20') & 0 & 0 & $\begin{array}{r}306.2\left(306.2,{ }^{\mathrm{a}} 307.0,^{\mathrm{b}}\right. \\
\left.307.2,{ }^{\mathrm{c}} 306.4^{\mathrm{d}}\right)\end{array}$ & $\begin{array}{c}0.75\left(0.78,^{\mathrm{a}} 0.79,^{\mathrm{b}}\right. \\
\left.1.056^{\mathrm{c}}\right)\end{array}$ \\
\hline & Stockmayer (eq 20) & 0 & 0 & 306.5 & 0.80 \\
\hline & Koningsveld et al. (eq 3, 4) & 0.623 & 0.308 & 305.2 & 0.29 \\
\hline & & $0.623^{\mathrm{e}}$ & $0.290^{\mathrm{e}}$ & 305.6 & 0.30 \\
\hline & Kamide-Matsuda: Phase separation & $0.6^{\mathrm{n}}$ & $0^{\mathrm{n}}$ & 306.4 & 0.35 \\
\hline & (eq 14-16) Cloud point & $0.643^{\mathrm{f}}$ & $0.200^{f}$ & 306.6 & 0.27 \\
\hline & Critical point & $0.623^{\mathrm{e}}$ & $0.290^{\mathrm{e}}$ & 305.6 & 0.27 \\
\hline & Critical point & 0.642 & 0.190 & 305.1 & 0.27 \\
\hline \multirow{5}{*}{$\begin{array}{l}\text { Second } \\
\text { virial } \\
\text { coefficient }\end{array}$} & Membrane osmometry & - & - & $307.6^{\mathrm{g}}$ & $0.36^{\mathrm{g}}$ \\
\hline & & - & - & $307.6^{\mathrm{h}}$ & $0.23^{\mathrm{h}}$ \\
\hline & Light scattering & - & - & $308.4^{\mathrm{i}}$ & $0.19 \pm 0.05^{\mathrm{i}}$ \\
\hline & & - & - & $307.0^{\mathrm{j}}$ & $0.36^{\mathrm{k}}$ \\
\hline & & - & - & $307.4^{1}$ & $0.18^{\mathrm{m}}$ \\
\hline
\end{tabular}

${ }^{\mathrm{a}}$ ref $11 ;{ }^{\mathrm{b}} \operatorname{ref} 15 ;{ }^{\mathrm{c}} \operatorname{ref} 2 ;{ }^{\mathrm{d}} \operatorname{ref} 11 ;{ }^{\mathrm{e}} \operatorname{ref} 8 ;{ }^{\mathrm{f}} \operatorname{ref} 9 ;{ }^{\mathrm{g}} \operatorname{ref} 3 ;{ }^{\mathrm{h}} \operatorname{ref} 11 ;{ }^{\mathrm{i}}$ ref $16 ;{ }^{\mathrm{j}}$ ref $17 ;{ }^{\mathrm{k}}$ calculated using $\theta\left(\partial A_{2} / \partial T\right)_{\theta}$ data in ref $17 ;{ }^{1}$ ref $20 ;{ }^{\mathrm{m}}$ calculated using $\theta\left(\partial A_{2} / \partial T\right)_{\theta}$ data in ref $20 ;{ }^{\mathrm{n}}$ ref 10 .

Table III. Flory temperature $\theta$ and entropy parameter $\psi$ of the lower solution critical point for the polystyrene-cyclohexane system

\begin{tabular}{|c|c|c|c|c|}
\hline Method & $p_{1}$ & $p_{2}$ & $\theta / K$ & $\psi$ \\
\hline \multicolumn{5}{|l|}{ (i) Critical point } \\
\hline Shultz-Flory (eq 20') & 0 & 0 & $486.6\left(486.0^{\mathrm{a}}\right)$ & $-1.20\left(-1.19^{\mathrm{a}}\right)$ \\
\hline Stockmayer (eq 20) & 0 & 0 & 486.8 & -1.20 \\
\hline Koningsveld et al. (eq 3, 4) & 0.571 & -0.047 & 487.5 & -0.61 \\
\hline Kamide-Matsuda: Phase separation & $0.60^{\mathrm{b}}$ & $0^{\mathrm{b}}$ & 487.6 & -0.52 \\
\hline Critical point & 0.602 & -0.347 & 487.2 & -0.42 \\
\hline
\end{tabular}

${ }^{a}$ ref $15 ;{ }^{b}$ ref 10.

and $v_{\mathrm{p}}^{\mathrm{c}}$ into eq 23 and ploting them as a function of $T_{\mathrm{c}}{ }^{-1}$ in Figure 2. $g_{00}$ was determined as 0.213 and $g_{01}$ as 151.7 for UCSP and $g_{00}=1.2447$ and $g_{01}=-297.0$ were obtained for LCSP. Using these $g$ functions, $p_{1}$, $p_{2}$ (both at $\theta$ ), $\theta$, and $\psi$ were evaluated as summarized in Tables II and III.

Figure 3 shows the plot of $v_{\mathrm{p}}^{\mathrm{c}}(\exp ) v s$. $v_{\mathrm{p}}^{\mathrm{c}}$ (theo) calculated for some typical combinations of $p_{1}$ and $p_{2}$ for the UCSP and LCSP of a $\mathrm{PS} / \mathrm{CH}$ system. In this figure the same data as those in Figures 1 and 2 were utilized. Figure 4 shows the relations among $p_{1}, p_{2}$ and $\delta$ for the PS/CH system. The most reasonable combination of $p_{1}$ and $p_{2}$, evaluated from Figure 4, is $p_{1}=0.642$ and $p_{2}=0.190$ for UCSP and $p_{1}=$ 0.602 and $p_{2}=-0.347$ for LCSP (Tables II and III).

The plots of $1 / T_{\mathrm{c}}$ against $X_{\mathrm{o}}^{\mathrm{c}}$ for the PS/CH system are shown in Figure 5. The results are summarized in Tables II and III, in which $\theta$ and $\psi$ values estimated originally in literature are shown in parenthesis for comparison. Krigbaum and his coworkers, ${ }^{3,11,16}$ and Schulz and Baumann ${ }^{17}$ determined the $\theta$ and $\psi$ of UCSP for the PS/CH system from the tem- 


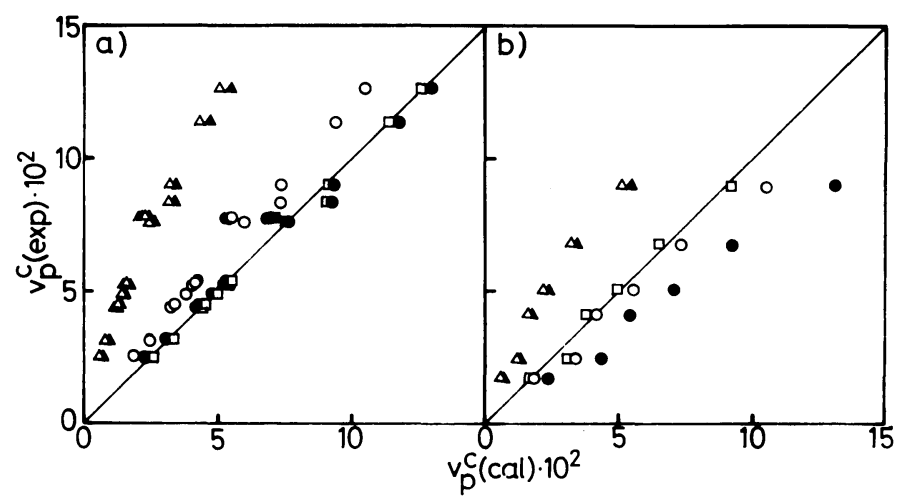

Figure 3. The critical concentration, experimentally determined, $v_{\mathrm{p}}^{\mathrm{c}}$ (exp) plotted against the critical concentration, theoretically calculated, $v_{\mathrm{p}}^{\mathrm{c}}$ (theo) for the UCSP (a) and LCSP (b) of the polystyrene/ cyclohexane system. $\triangle$, Shultz-Flory ${ }^{2}$ [eq 19']; $\boldsymbol{\Delta}$, Stockmayer ${ }^{4}$ [eq 19]; $\bigcirc$, Kamide-Matsuda [eq 14, 15, $p_{1}=0.6, p_{2}=0$ ]; $\bigcirc$, Kamide-Matsuda [eq 14, 15, $p_{1}=0.623, p_{2}=0.290^{8}$ ]; $\square$, Kamide-Matsuda [eq 14, 15, $p_{1}=0.642, p_{2}=0.190$ for UCSP and $p_{1}=0.602, p_{2}=-0.347$ for LCSP].

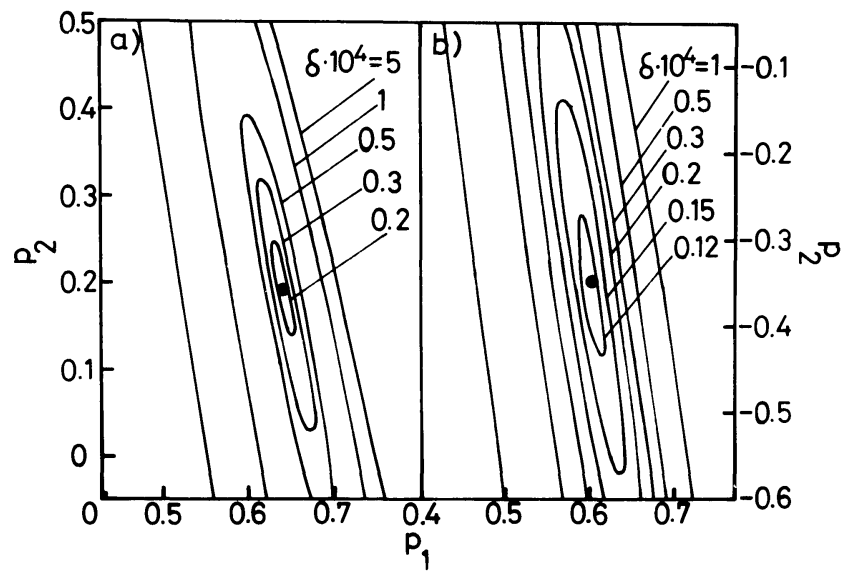

Figure 4. Relations among $p_{1}, p_{2}$ and $\delta$ for the UCSP (a) and LCSP (b) of the polystyrene/cyclohexane system. Number on curve denotes $\delta \times 10^{4}$. Filled mark corresponds to the most ideal combination of $p_{1}$ and $p_{2}$ to describe the critical solution point.

perature dependence of the second virial coefficient near $\theta$ temperature. The literature data show variation in $\psi$ from 0.18 to 0.36 and in $\theta$ from 307.0 to $308.4 \mathrm{~K}$, as listed in Table II.

\section{b) Atactic Polystyrene(PS)-Methylcyclo-} hexane (MCH)

Kamide et al. determined $p_{1}=0.7$ and $p_{2} \simeq 0$ by phase separation in the UCSP region ${ }^{10}$ and $p_{1}=0.78$ and $p_{2}=0.33$ by osmotic pressure. ${ }^{18}$ Dobashi and his coworkers ${ }^{19}$ estimated UCSP as the intercept of the co-existence curve and its diameter for PS samples with $X_{w} / X_{n}<1.06$ and obtained $p_{1}=0.604$ and $p_{2}=0.299$ at $344 \mathrm{~K}$ using Koningsveld et al.'s method. ${ }^{8}$

Here, we analyzed the literature data of the UCSP of Dobashi et al. (9 samples) $)^{19}$ and the UCSP and LCSP of Saeki and his coworkers (6 samples) ${ }^{15} . X_{z}$ was calculated assuming the SZ distribution for all samples.

Figure 6 shows a plot of $Y$ vs. $v_{\mathrm{p}}^{\mathrm{c}}$ for $\mathrm{PS} / \mathrm{MCH}$ system. A comparison of the re- 


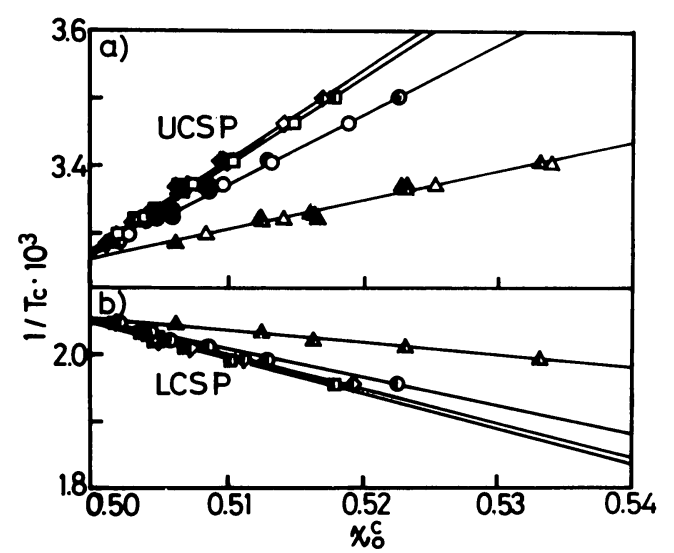

Figure 5. $1 / T_{\mathrm{c}}$ plotted against $\chi_{0}^{\mathrm{c}}$ for the UCSP (a) and LCSP (b) of the polystyrene/cyclohexane system. Triangle, Schultz-Flory plot [eq 21']; circle, KamideMatsuda plot [eq 14, 15, $p_{1}=0.6, p_{2}=0$ ]; rectangle, Kamide-Matsuda plot [eq 14, 15, $p_{1}=0.623, p_{2}=$ $0.290^{8}$ ]; diamond Kamide-Matsuda plot [eq 14, 15, $p_{1}=$ $0.642, p_{2}=0.190$ for UCSP and $p_{1}=0.602, p_{2}=-0.347$ for LCSP]; unfilled mark, data of Koningsveld et al. ${ }^{12}$; filled mark, data of Kuwahara et al. ${ }^{13}$; half-filled mark, data of Saeki et al. ${ }^{15}$

lations between $Y$ and $v_{\mathrm{p}}^{\mathrm{c}}$ with eq 22 yields $g_{1}=$ $0.219_{2}$ and $g_{2}=0.060_{1}$ for UCSP and $g_{1}=$ -0.007 and $g_{2}=-0.167$ for LCSP.

Figure 7 shows $g_{0}$ plotted against $1 / T_{\mathrm{c}}$ for the same system, we obtain $g_{00}=1.053$ and $g_{01}=-273.4$ for LCSP. The $p_{1}, p_{2}$, (both at $\theta), \theta$ and $\psi$ values calculated from $g$ function are listed in Tables IV and V.

Figure 8 shows plots of $v_{\mathrm{p}}^{\mathrm{c}}(\exp )$ against $v_{\mathrm{p}}^{\mathrm{c}}$ (theo), calculated, assuming various values of $p_{1}$ and $p_{2}$, for PS/MCH system.

Figure 9 shows the relations among $p_{1}, p_{2}$, and $\delta$ for the UCSP and LCSP of the PS/MCH system. From this figure, we obtain the most reasonable combination $p_{1}=0.602$ and $p_{2}=$ 0.234 for UCSP and $p_{1}=0.649$ and $p_{2}=$ -1.183 for LCSP.

Figure 10 shows $1 / T_{\mathrm{c}}$ plotted as a function of $\chi_{0}^{\mathrm{c}}$ for the PS/MCH system. From the intercept and slope of the plot $\theta$ and $\psi$ were evaluated and summarized in Tables IV and V.

Schulz and Baumann ${ }^{17}$ determined $\theta$ to be $68^{\circ} \mathrm{C}$ and $\theta\left(\partial A_{2} / \partial T\right)_{\theta}$ to be $2.16 \times 10^{-3} \mathrm{~cm}^{3}$

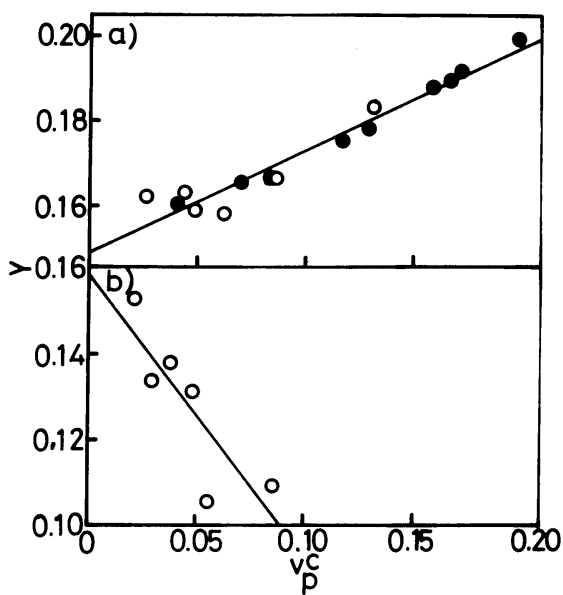

Figure 6. Plot of $Y$ in eq 21 against $v_{\mathrm{p}}^{\mathrm{c}}$ for the UCSP (a) and LCSP (b) of the polystyrene/methylcyclohexane system. $\bigcirc$, Saeki et al. ${ }^{15}$; , Dobashi et al. ${ }^{19}$

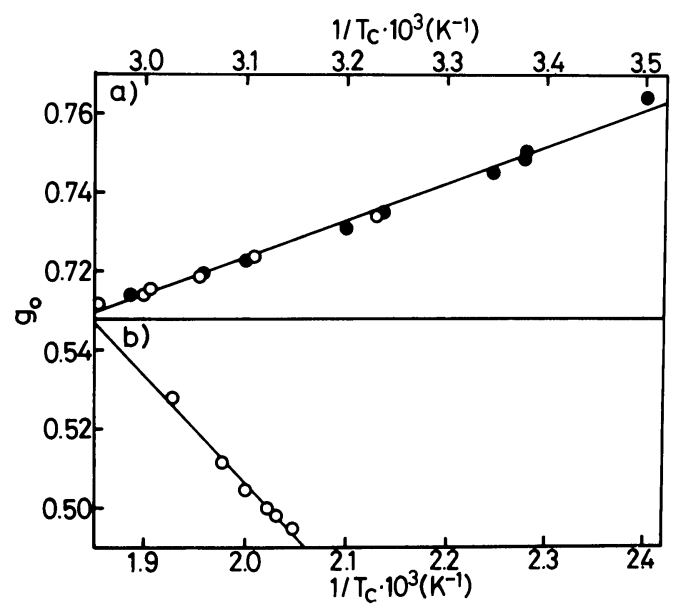

Figure 7. Plot of $g_{0}$ in eq 22 versus $1 / T_{\mathrm{c}}$ for the UCSP (a) and LCSP (b) of the polystyrene/methylcyclohexane system. $\bigcirc$, Saeki et al. ${ }^{15} ; 0$, Dobashi et al. ${ }^{19}$

$\mathrm{g}^{-2}$ for UCSP by light scattering method. $\psi$ was thus calculated by the following equation

$$
\psi=\left(V_{1} / \bar{v}^{2}\right) \theta\left(\partial A_{2} / \partial T\right)_{\theta}
$$

as 0.30 . Here, $\left(\partial A_{2} / \partial T\right)_{\theta}$ is the temperarure dependence of $A_{2}, V_{1}$, the molar volume of solvent $\left(136.4 \mathrm{~cm}^{3} \mathrm{~mol}^{-1}\right)$ and $\bar{v}$, the specific volume of the polymer $\left(0.99 \mathrm{~cm}^{3} \mathrm{~g}^{-1}\right)$. Kotera et $a .^{21}$ also evaluate-independently $\theta$ and $\psi$ for 


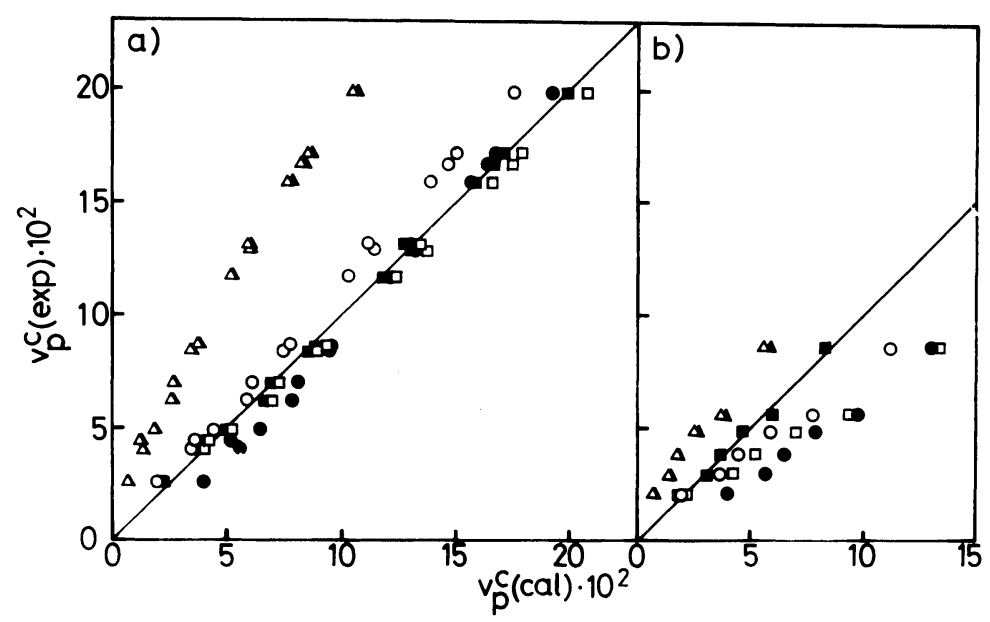

Figure 8. The critical concentration, experimentally determined, $V_{\mathrm{p}}^{\mathrm{c}}(\exp )$ plotted against the critical concentration, theoretically calculated $v_{\mathrm{p}}^{\mathrm{c}}$ (theo) for the UCSP (a) and LCSP (b) of the polystyrene/ methylcyclohexane system. $\triangle$, Shultz-Flory [eq 19']; $\boldsymbol{\Delta}$, Stockmayer [eq 19]; $\bigcirc$, Kamide-Matsuda [eq 14, $15, p_{1}=0.6, p_{2}=0$ ]; $\bigcirc$, Kamide-Matsuda [eq 14, 15, $p_{1}=0.7, p_{2}=0$ ]; $\square$, Kamide-Matsuda [eq 14, 15, $p_{1}=$ $0.604, p_{2}=0.299^{19}$ ]; $\square$, Kamide-Matsuda [eq 14, 15, $p_{1}=0.602, p_{2}=0.234$ for UCSP and $p_{1}=0.649, p_{2}=$ -1.183 for LCSP].

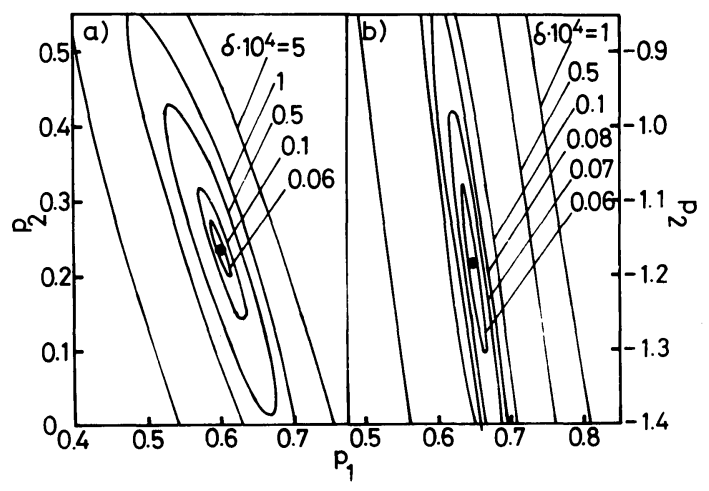

Figure 9. Relations among $p_{1}, p_{2}$, and $\delta$ for the UCSP (a) and LCSP (b) of the polystyrene/cyclohexane system. Number on curve denotes $\delta \times 10^{4}$. Filled mark corresponds to the most ideal combination of $p_{1}$ and $p_{2}$ to describe the critical solution point.

UCSP using the same method. The results are in Table IV.

\section{DISCUSSION}

It is evident from Figures 3 and 8 that the Shultz-Flory and Stockmayer theories significantly underestimate $v_{\mathrm{p}}^{\mathrm{c}}: v_{\mathrm{p}}^{\mathrm{c}}(\exp )>v_{\mathrm{p}}^{\mathrm{c}}($ theo $)$

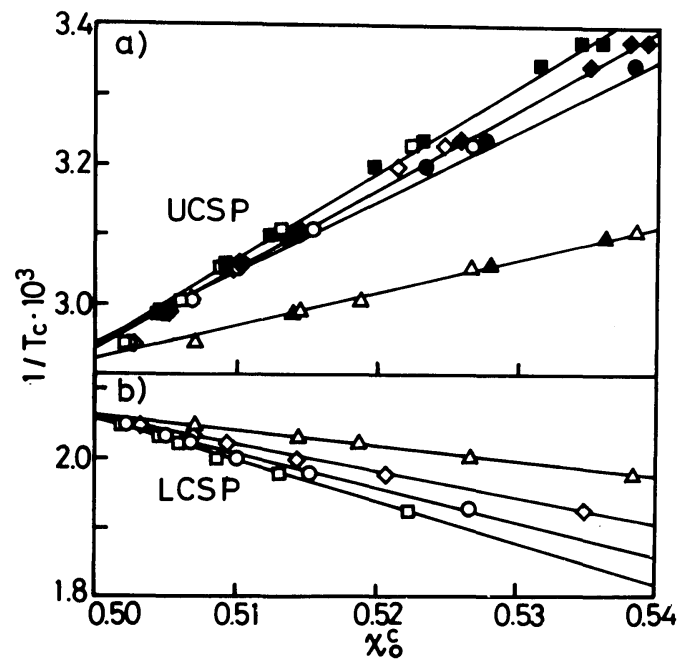

Figure 10. $1 / T_{\mathrm{c}}$ plotted against $\chi_{0}^{\mathrm{c}}$ for the UCSP (a) and LCSP (b) of the polystyrene/methylcyclohexane system. Triangle, Shultz-Flory plot [eq 21']; circle, Kamide-Matsuda plot [eq 14, 15, $p_{1}=0.7, p_{2}=0$ ]; rectangle, Kamide-Matsuda plot [eq 14, 15, $p_{1}=0.604$, $p_{2}=0.299$ ]; diamond Kamide-Matsuda plot [eq 14, 15, $p_{1}=0.602, p_{2}=0.234$ for UCSP and $p_{1}=0.649, p_{2}=$ -1.183 for LCSP]; unfilled mark, data of Saeki et al. ${ }^{15}$; filled mark, data of Dobashi et al. ${ }^{19}$

and that consideration of the simple parameter $p_{1}$ in Kamide-Matsuda's method greatly re- 
Table IV. Flory temperature $\theta$ and entropy parameter $\psi$ of the upper solution critical point for the polystyrene-methylcyclohexane system

\begin{tabular}{|c|c|c|c|c|c|c|}
\hline \multicolumn{3}{|c|}{ Method } & \multirow{2}{*}{$\begin{array}{l}p_{1} \\
0\end{array}$} & \multirow{2}{*}{$\begin{array}{l}p_{2} \\
0\end{array}$} & \multirow{2}{*}{$\frac{\theta / K}{342.3\left(344^{\mathrm{a}}\right)}$} & \multirow{2}{*}{$\frac{\psi}{0.61\left(0.56^{\mathrm{a}}\right)}$} \\
\hline \multirow{7}{*}{ Critical point } & \multicolumn{2}{|l|}{ Shultz-Flory (eq 20') } & & & & \\
\hline & \multicolumn{2}{|l|}{ Stockmayer (eq 20) } & 0 & 0 & 342.3 & 0.61 \\
\hline & \multirow{2}{*}{\multicolumn{2}{|c|}{ Koningsveld et al. (eq 3, 4) }} & 0.602 & 0.363 & 339.6 & 0.27 \\
\hline & & & $0.604^{\mathrm{b}}$ & $0.299^{b}$ & 343.4 & - \\
\hline & \multirow{3}{*}{$\begin{array}{l}\text { Kamide-Matsuda: } \\
\quad(\text { eq } 14-16)\end{array}$} & Phase separation & $0.70^{\mathrm{c}}$ & $0^{c}$ & 335.7 & 0.24 \\
\hline & & Coexistence curve & $0.604^{b}$ & $0.299^{\mathrm{b}}$ & 340.4 & 0.23 \\
\hline & & Critical point & 0.602 & 0.234 & 340.2 & 0.25 \\
\hline $\begin{array}{l}\text { Second virial } \\
\text { coefficient }\end{array}$ & \multicolumn{2}{|l|}{ Light scattering } & - & - & $\begin{array}{l}341^{\mathrm{d}} \\
340.4^{\mathrm{f}}\end{array}$ & $\begin{array}{l}0.30^{\mathrm{e}} \\
0.16^{\mathrm{g}}\end{array}$ \\
\hline
\end{tabular}

${ }^{\mathrm{a}}$ ref $15 ;{ }^{\mathrm{b}}$ ref $19 ;{ }^{\mathrm{c}}$ ref $10 ;{ }^{\mathrm{d}}$ ref $17 ;{ }^{\mathrm{e}}$ calculated using the $\theta\left(\partial A_{2} / \partial T\right)_{\theta}$ data in ref $17 ;{ }^{\mathrm{f}}$ ref $20 ;{ }^{\mathrm{g}}$ calculated using the $\theta\left(\partial A_{2} / \partial T\right)_{\theta}$ data in ref 20 .

Table V. Flory temperature $\theta$ and entropy parameter $\psi$ of the lower solution critical point for polystyrene-methylcyclohexane

\begin{tabular}{|c|c|c|c|c|}
\hline Method & $p_{1}$ & $p_{2}$ & $\theta / K$ & $\psi$ \\
\hline \multicolumn{5}{|l|}{ (i) Critical point } \\
\hline Shultz-Flory (eq 20') & 0 & 0 & $485.1\left(484^{a}\right)$ & $-0.96\left(-0.94^{\mathrm{a}}\right)$ \\
\hline Stockmayer (eq 20) & 0 & 0 & 485.1 & -0.96 \\
\hline Koingsveld et al. (eq 3, 4) & 0.643 & $-1.00_{8}$ & 487.8 & -0.56 \\
\hline Kamide-Matsuda: Phase separation & $0.7^{\mathrm{b}}$ & $0^{\mathrm{b}}$ & 489.5 & -0.31 \\
\hline Critical point & 0.649 & -1.183 & 487.9 & -0.54 \\
\hline
\end{tabular}

${ }^{a}$ ref $15 ;{ }^{b}$ ref 10 .

duces the disagreement between $v_{\mathrm{p}}^{\mathrm{c}}(\exp )$ and $v_{\mathrm{p}}^{\mathrm{c}}($ theo $)$. However, usage of the single parameter $p_{1}$ is not sufficient to cover a wide range of $v_{\mathrm{p}}^{\mathrm{c}}$. For example, $v_{\mathrm{p}}^{\mathrm{c}}$ (theo), calculated using $p_{1}=0.6$ for the UCSP of the PS/MCH system, agrees well with $v_{\mathrm{p}}^{\mathrm{c}}(\exp )$, only at the lowest $X_{w}$ fraction and its difference with $v_{\mathrm{p}}^{\mathrm{c}}$ (theo) increases linearly and that between $v_{\mathrm{p}}^{\mathrm{c}}$ (theo), calculated with $p_{1}=0.7$, and $v_{\mathrm{p}}^{\mathrm{c}}(\exp )$ increases with decreasing $v_{\mathrm{p}}^{\mathrm{c}}$ (theo).

Not only $p_{1}$, but also $p_{2}$ are necessary and sufficient to describe the $v_{\mathrm{p}}^{\mathrm{c}}$ of polymer solutions as in the case of the cloud point curve. ${ }^{9}$ Note that the phase separation characteristics of the two phase equilibrium can be approximately explained by only considering $p_{1}$ and the effect of $p_{2}$ on these are insignificant, probably due to the large error involved in the separation experiments.
Three procedures are available to determine $p_{1}$ and $p_{2}$ concurrently, from the critical solution point ( $v_{\mathrm{p}}^{\mathrm{c}}$ and $T_{\mathrm{c}}$ ) (Koningsveld et al. . $^{8,12}$ and Kamide and Matsuda) or cloud point curve (Kamide et al. ${ }^{9}$ ). Previous work by Kamide et al. ${ }^{9}$ on the cloud point curve in the UCSP region for the $\mathrm{PS} / \mathrm{CH}$ system yields results on $p_{1}$ and $p_{2}$ throughly consistent with those of the present study: $p_{1}=0.64$ and $p_{2}=$ 0.19 were estimated for the UCSP of the $\mathrm{PS} / \mathrm{CH}$ system and Tables $\mathrm{II}-\mathrm{V}$ show Koningsveld et al.'s method and KamideMatsuda's method, both based on the critical solution point, to yield almost the same $p_{1}$ for the UCSP and LCSP of the the polymer/ solvent systems investigated, but the difference in $p_{2}$ obtained by two methods is remarkable. The plot of $Y$ against $v_{\mathrm{p}}^{\mathrm{c}}$ scatters considerably (see, Figures 1 and 6) and the correlationships 
$r$ between them are not satisfactorily high, for example, $r=0.87$ and -0.80 for the UCSP and LCSP of PS/CH system. The slope of the plots give $4 g_{2}$ and relatively larger uncertainty in the estimated value of $g_{2}$ may cause this pronounced scattering. The situation is almost the same even in the Kamide-Matsuda method but the $p_{2}$ value is also rather less accurate (see, Figures 4 and 9). These low degrees of accuracy in estimating $p_{2}$ in both methods can explain the noticeable variation in $p_{2}$ values. As will be demonstrated latter, since the contribution of $p_{2}$ to $\theta$ and $\psi$ is fortunately relatively small, the scattering of $p_{2}$ is not a serious problem.

In this paper, we assumed $p_{1}$ and $p_{2}$ to be temperature-independent over a narrow temperature range. But this assumption may hold for a very limited extent over a wide temperature range for both UCSP and LCSP. However, for the UCSP and LCSP regions of the $\mathrm{PS} / \mathrm{CH}$ and $\mathrm{PS} / \mathrm{MCH}$ systems, $p_{1}$ was found to be $0.6 \pm 0.04$ by the Kamide-Matsuda method. This does not means that $p_{1}$ is constant over a wide temperature range since there is the possibility that $p_{1}$ not in the theta region deviates from that in the UCSP and LCSP regions. For these systems, $p_{2}$ is always positive in the UCSP region, but negative in the LCSP region.

All the Shultz-Flory, Stockmayer and Kamide-Matsuda plots (Figures 5 and 10), though the former two underestimate $v_{\mathrm{p}}^{\mathrm{c}}$ significantly, give very straight lines between $T_{\mathrm{c}}$ and $\chi_{0}^{\mathrm{c}}$ and thus they cannot be evaluated simply on the basis of their linearity. These plots have an approximately common intercept at $\chi_{0}^{\mathrm{c}}(=0.5)$ so that the $\theta$ value obtained by these methods is roughly constant, regardless of the analytical method employed, for a given polymer/solvent system. A detailed investigation showed that for the UCSP of the PS/CH and PS/MCH systems, in which both $p_{1}$ and $p_{2}$ are positive, the Shultz-Flory and Stockmayer methods overestimate $\theta$ by a few degrees, but for the LCSP of these systems in which $p_{1}>0$ and $p_{2}<0$, these methods underestimate $\theta$ slightly. For all the systems, the Koningsveld et al. procedure and Kamide-Matsuda method give almost the same values of $\theta$, within $\pm 0.1-$ $0.3 \mathrm{~K}$. The value of $\theta$, estimated by the second virial coefficient method, is about $2.5 \mathrm{~K}$ higher than that either by the Koningsveld et al. or Kamide-Matsuda method for the PS/ $\mathrm{CH}$ system and is very near to that estimated by the Shultz-Flory method. On the other hand, for the PS/MCH system, the value of $\theta$ obtained by the second virial coefficient method agrees fairly well with those by the Koningsveld et al. method and KamideMatsuda methods. Up to now, the ShultzFlory method has been used most for estimating $\theta$ as compiled in the Polymer Handbook $^{21}$ from the critical point data and $\theta$ temperatures estimated thus may have an experimental uncertainty of \pm a few degree, even if monodisperse polymer samples are used.

As long as the polymolecularity of the polymer samples is small, $\left(X_{w} / X_{n}<1.1\right)$ as in the case of the vast majority samples used for analysis of $\mathrm{PS} / \mathrm{CH}$ and $\mathrm{PS} / \mathrm{MCH}$ systems, and/or the critical solution points are determined directly as points at which the two phase volume ratio is unity as is the case of Koningsveld et al.'s data ${ }^{12}$ for the UCSP of the $\mathrm{PS} / \mathrm{CH}$ system, there is no significant difference in $\theta$ value, as the theory predicts, between Shultz-Flory and Stockmayer method.

For polystyrene solutions, the absolute magnitude of $\psi$ values estimated by the methods of Koningsveld et al. and Kamide and Matsuda are only half or $1 / 3$ those by the Shultz-Flory and Stockmayer methods. The latter two methods give the same $\psi$, which is usually very large compared with that by the second virial coefficient method. The KamideMatsuda method yields a slightly smaller $\psi$ (by -0.02) than that of Koningsveld et al. $\psi$ estimated for the UCSP of PS/CH by the second virial coefficient method varies from $0.18-0.36$ and averages 0.264 , which agrees 
fairly well with $\psi=0.27$ obtained by the Kamide-Matsuda method. For the UCSP of $\mathrm{PS} / \mathrm{MCH}, \psi$ was independently determined as 0.30 and 0.16 from the temperature dependence of the second virial coefficient. Their average value $(0.23)$ was near to 0.25 as estimated by the Kamide-Matsuda method. Hence, as far as a non-polar polymer solution is concerned, $\psi$ values estimated so far by the Shultz-Flory method, are erroneously overestimated owing to the neglect of the concentration dependence of the $\chi$-parameter.

From the above, it is clear that the great difference between $\psi$ values obtained from critical points according to the Shultz-Flory procedure and second virial coefficient is evidently not due to the effect of the polydispersity of the polymer on the critical point, but almost entirely to ignorance of the concentration dependence of the $\chi$-parameter $\left(p_{1}\right.$ and $p_{2}$ ) in the Shultz-Flory method. The second virial coefficient corresponds to the term containing $v_{\mathrm{p}}{ }^{2}$ when the chemical potential of the solvent in a solution is expanded in a polynomial of $v_{\mathrm{p}}$ and therefore $A_{2}$ corresponds to $\chi_{0}$, a concentration-independent term of the $\chi$ parameter in eq 5. Therefore, the existence of the concentration dependence of $\chi$ has no effect on the results obtained using eq 25 , and in this sense, the second virial coefficient method is unconditionally preferable from a theoretical point of view.

The effect of $p_{1}$ and $p_{2}$ on the $\psi$ parameter were examined for the $\mathrm{PS} / \mathrm{CH}$ system in Figures $1-5$. The results are shown in Figure 11. $\psi$ decreases with an increase in $p_{1}$ and $p_{2}$, but the former plays a predominant role and the latter is usually negligible. It is thus confirmed that the relatively low reliability of $p_{2}$ causes no significant variation in $\psi$. The variation in $p_{1} \pm 0.1$ brings about a variation in $\psi \pm 0.05$, which is too large to neglect. Therefore, to estimate $\psi$ accurately, $p_{1}$ should have a precision at least within \pm 0.05 .

The most reasonable values of $p_{1}$ and $p_{2}$, evaluated for $\mathrm{PS} / \mathrm{CH}$ and $\mathrm{PS} / \mathrm{MCH}$, are sum-

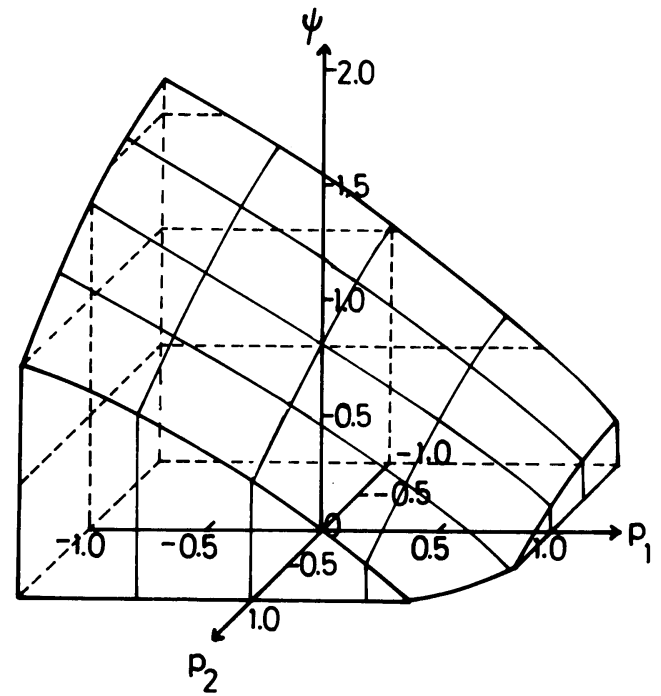

Figure 11. Effect of $p_{1}$ and $p_{2}$ on entropy parameter $\psi$ of UCSP for polystyrene/cyclohexane system. The same data in Figures $1-5$ were used for calculating $\psi$.

Table VI. Most ideal combination of $p_{1}$ and $p_{2}$ for a polymer-solvent system

\begin{tabular}{clllllll}
\hline \multirow{2}{*}{ Polymer } & Solvent & \multicolumn{2}{c}{ UCSP } & & \multicolumn{2}{c}{ LCSP } \\
\cline { 3 - 4 } \cline { 6 - 7 } & & $p_{1}$ & $p_{2}$ & & $p_{1}$ & $p_{2}$ \\
\hline Polystyrene & $\begin{array}{l}\text { Cyclohexane } \\
\text { Polystyrene }\end{array}$ & 0.64 & 0.19 & & 0.60 & -0.35 \\
& $\begin{array}{l}\text { Methyl- } \\
\text { cyclohexane }\end{array}$ & 0.60 & 0.23 & & 0.65 & -1.18 \\
\hline
\end{tabular}

marized in Table VI. No possible explanation for the negative $p_{2}$ in the LCSP region is offered and this matter is open for further study.

In summary,

1) For a reasonably accurate determination of the critical solution point, two concentration dependence parameters $p_{1}$ and $p_{2}$ should be carefully taken into account.

2) By comparing the observed critical solution concentration $v_{\mathrm{p}}^{\mathrm{c}}(\exp )$ with the theoretical concentration $v_{\mathrm{p}}^{\mathrm{c}}$ (theo), calculating by eq 14 and 15 with assumed values of $p_{1}$ and $p_{2}$, we can determine $p_{1}$ and $p_{2}$ simultaneously.

3) The $p_{1}$ value obtained thus for the PS/ $\mathrm{CH}$ system is in good agreement with that by 
the Kamide et al. method, using the cloud point curve and that by Koningsveld et al. method, using the same critical solution data in each case.

4) Some large disagreement was observed between the $p_{2}$ values estimated by our method, and those by Koningsveld et al.'s method, using the same critical solution data in either case.

5) $\psi$ as well as $\theta$ can be accurately determined by measuring $T_{\mathrm{c}}$ for polymer samples with known $X_{w}$ and $X_{z}$ and using $p_{1}$ and $p_{2}$ determined from the same critical point data or the cloud point curve.

6) If $p_{1}$ and $p_{2}$ are known in advance, the new procedure is undoubtedly much more straight forward compared to the Koningsveld et al. method. The latter method requires $v_{\mathrm{p}}^{\mathrm{c}}$ in addition to $T_{\mathrm{c}}$.

7) $\psi$ obtained from the critical point for the $\mathrm{PS} / \mathrm{CH}$ and $\mathrm{PS} / \mathrm{MCH}$ systems agrees with that calculated by the second virial coefficient, provided, in the former, $p_{1}$ and $p_{2}$ are reasonably taken into account.

\section{REFERENCES}

1. See, for example, P. J. Flory, "Principle of Polymer Chemistry," Cornell Univ. Press, Ithaca, N.Y., 1953.

2. A. R. Shultz and P. J. Flory, J. Am. Chem. Soc., 74, 4760 (1952).
3. W. R. Krigbaum, J. Am. Chem. Soc., 76, 3758 (1954).

4. W. H. Stockmayer, J. Chem. Phys., 17, 588 (1949).

5. See, for example, M. Kurata, "Thermodynamics of Polymer Solutions," Harwood Academic Pubs., Chur, London, New York, 1982, Chapter 2.

6. K. Kamide and Y. Miyazaki, Polym. J., 13, 325 (1981).

7. K. Kamide, T. Abe, and Y. Miyazaki, Polym. J., 14, 355 (1982).

8. R. Koningsveld and L. A. Kleintjens, Macromolecules, 5, 637 (1971).

9. K. Kamide, S. Matsuda, T. Dobashi, and M. Kaneko, Polym. J., 16, 821 (1984).

10. K. Kamide, Y. Miyazaki, and T. Abe, Makromol. Chem., 177, 485 (1976).

11. W. R. Krigbaum and D. O. Geymer, J. Am. Chem. Soc., 81, 1859 (1959).

12. R. Koningsveld, L. A. Kleintjens, and A. R. Shultz, J. Polym. Sci., A-2, 8, 1261 (1970).

13. N. Kuwahara, N. Nakata, and M. Kaneko, Polymer, 14, 415 (1973).

14. Th. G. Scholte, J. Polym. Sci., A-2, 8, 841 (1970).

15. S. Saeki, N. Kuwahara, S. Konno, and M. Kaneko, Macromolecules, 6, 246 (1973).

16. W. R. Krigbaum and D. K. Carpenter, J. Phys. Chem., 59, 1166 (1959).

17. G. V. Schulz and H. Baumann, Makromol. Chem., 60, 120 (1963).

18. K. Kamide, K. Sugamiya, T. Kawai, and Y. Miyazaki, Polym. J., 12, 67 (1980).

19. T. Dobashi, M. Nakata, and M. Kaneko, J. Chem. Phys, 72, 15 (1980).

20. A. Kotera, T. Saito, and N. Fukisaki, Rep. Prog. Polym. Phys. J., VI, 9 (1963).

21. J. Bandrup and E. H. Immergut, Ed., "Polymer Handbook," 2nd ed, John Wiley \& Sons, Inc., New York, N. Y., 1974, Chapter IV, p 157. 